\title{
LIVER RESECTION FOR METASTASES FROM COLORECTAL CANCER
}

\author{
Brig HG MUKHOPADHYAY, sM ", Lt Col LS VOHRA ${ }^{+}$, \\ Lt Col AK SHARMA ${ }^{\#}$, Dr R SOLANKI ${ }^{* *}$, \\ Col PS REDDY $^{++}$, Lt Col GS CHOPRA ${ }^{\#}$, \\ Brig P SUBHAS vSM
}

\begin{abstract}
Five percent of patients with liver secondaries from colorectal carcinoma are potentially resectable and several studies have demonstrated significantly improved survival following resection. Two hundred and ten patients operated for colorectal carcinoma were followed up. Computed tomography confirmed potentially resectable metastasis to the liver in 38 . On exploration 18 patients who had 4 or less hepatic metastases and no extrahepatic disease, underwent resection of their secondaries. Fourteen were males and 4 females with a mean age of 43.5 (SD 13.6, range 18-72) years. Ten patients presented with synchronous liver metastasis and 8 had metachronous disease. There was no post-operative mortality. All 18 have been followed up. for a median period of 23.5 (range 12-38) months. Seven patients are alive and well with no evidence of recurrence at a median period of 28 months (survival $39 \%$ ). Four are alive with local recurrence in the liver. Median time to recurrence was 22 months. Seven patients have died of disseminated disease. The disease free survival at 28 months is $39 \%$ and the overall survival $61 \%$. A close follow-up protocol for all patient undergoing curative surgery for colorectal cancer is essential, if such patients are to be selected early.
\end{abstract}

MJAFI 1999; 55 : 9-12

KEYWORDS: Colorectal cancer; Liver resection; Metastases.

\section{Introduction}

$\mathbf{T}$

The incidence of synchronous liver secondaries from colorectal carcinoma is $15-30 \%$, and a similar percentage of patients develop metachronous metastases, usually within three years $[1,2]$.Liver metastasis in these patients limits survival to months if they have a heavy tumour burden [3]. However, $5 \%$ of such patients are potentially resectable and several studies have demonstrated significantly improved survival following resection $[4,5]$. Techniques of hepatic resection have greatly evolved since it was introduced as a viable procedure in 1980 , and an operative mortality of less than $5 \%$ strongly justifies such resections [4].

We present a retrospective analysis of our experience with liver resections of secondaries from colorectal malignancy over the past three years.

\section{Patients and Methods}

In our centre patients operated for colorectal cancer are kept on a three monthly follow-up for two years, followed by a 6 monthly follow-up for the next three years. We have followed 210 such patients since 1993. Of these 156 underwent resection of the primary tumour in our centre and another 54 had been operated at other hospitals, and referred for follow-up/adjuvant treatment. Serial liver function test, chest X-ray, carcinoembryonic antigen (CEA) and ultrasound scans (US) were performed in all. A CEA of $3 \mathrm{ng} / \mathrm{ml}$ or a suspicious area of altered echogenicity on US prompted further evaluation by colonoscopy and contrast enhanced computed tomography (CECT). CT guided FNAC confirmed potentially resectable metastasis of the liver in 38 patients who on CECT did not have evidence of extra-hepatic disease and were otherwise in good general health. These 38 patients underwent exploratory laparotomy to undergo a possible hepatic resection. On surgical exploration however, 20 (52\%) of these 38 were found to have extensive, previously unrecognised disease. Eighteen patients who had 4 or less hepatic metasis and no extrahepatic disease, underwent hepatic resection of their secondaries. Synchronous metastasis of $<2 \mathrm{~cm}$ or a metastasis localised to left lobe were resected in the same operation as the primary colorectal resection. Major liver resections were, however, carried out as a staged procedure, if a CECT 6 to 8 weeks after colonic resection did not reveal extensive disease.

The type of surgical resection carried out was determined by the location, size and number of metastasis. Nodule upto $2 \mathrm{~cm}$ in diameter were considered for a single or multiple wedge resections. Larger metatasis were resected by formal hepatectomy. A clearance of at least one $\mathrm{cm}$ was aimed at in all dimensions. Prophylactic lymph node clearance of the hepato-duodenal ligament was not carried out.

All patients received post-operative chemotherapy and are on three monthly follow-up.

\section{Results}

Of the 38 patients who underwent surgical exploration for a possible liver resection 27 were males and 11 females. The diagnosis of liver metastastis was suspected on US and raised CEA levels in $27(71 \%)$ and in $8(21 \%)$ on the basis of an elevated CEA

"Consultant (Surgery) ${ }^{+}$Classified Specialist (Surgical Oncology), ${ }^{*}$ Classified Specialist (GI Surgery), " Trainee Surgeon, ${ }^{++}$Senior

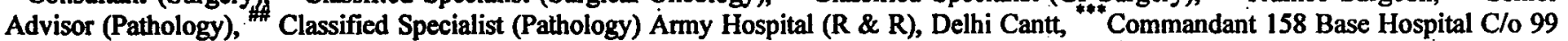
APO. 
alone. In another 3 patients, resectable liver metastasis were incidentally found on laparotomy for the primary colorectal surgery. CEA levels were found to be significantly elevated in $35(92 \%)$ of the 38 patients. In 3 patients however, metastatic liver disease was present despite normal levels of CEA. Serum alkaline phosphatase was found to be elevated in only $10(26 \%)$ of the 38 patients and did not correlate with the presence of an elevated CEA or liver metastasis.

Of the 18 who underwent hepatic resection, 14 were males and 4 females with a mean age of 43.5 (SD 13.6, range 18-72) years. Ten patients presented with synchronous liver metastasis and 8 were found to have metachronous disease after a mean interval of 3.5 (SD range 1.2-4) years since resection of the colorectal primary. None of the 18 patients had symptoms referable to the hepatic metastasis (Table 2).

Seven patients underwent a major liver resection (right hepatectomy in 3, and left hepatectomy in 4). Three patients underwent a left lobectomy and in 8 wedge resection of the liver was done. Four additional wedge resections were performed in combination with major resections of the left lobe (Table 1). Standard techniques of liver resection were employed and sophisticated equipment like ultrasonic dissectors, cryoprobes or lasers were not used. Blood transfusion was required only in major resections with a mean requirement of 3.3 (SD 2.8, range 1-9) units. The mean duration of postoperative hospital stay after major and minor resections was 32 (SD 10.7; range 20-58) and 12 (SD 3.8; range 9-6) days respectively. There was no post-operative mortality.

TABLE 1

Extent of surgery in 18 patients undergoing hepatic resection for colorectal secondaries

\begin{tabular}{lcc}
\hline Procedure & Synchronous & Metachronous \\
\hline Right hepatectomy & 2 & 1 \\
Left hepatectomy & $1^{*}$ & 3 \\
Left lobectomy & $1^{*}$ & $2^{*}$ \\
Wedge resections & 6 & 2 \\
\hline
\end{tabular}

* Combined with a wedge resection of the right lobe

All 18 patients have been followed up for a median period of 23.5 (range 12-38) months. Seven patients are alive and well with no evidence of recurrence at a median period of 28 months (survival $39 \%$ ). Four are alive with local recurrence in the liver. Median time to recurrence was 22 months. Seven patients have died of disseminated disease. Thus the disease free survival at 28 months was $39 \%$ and the overall survival $61 \%$.

\section{Discussion}

Numerous studies of the natural history of colorectal cancer have shown that although liver metastasis is the primary determinant of patient survival, it is most often not the only site of metastases in a diffuse picture of disseminated disease [6]. Patients with untreated liver metastasis have median survival rates of 6 to 18 months and the single most important factor for length of survival is the extent of liver involvement [7], with a mean survival of 3 months in patients with widespread bilateral metastasis as compared to 17 months in patients with multiple metastasis in one lobe
TABLE 2

Outcome of liver resection in 18 patients with metastatic colorectal cancer

\begin{tabular}{lllll}
\hline Surgical procedure & $\mathrm{n}$ & NED & Alive with disease & Deaths \\
\hline Right hepatectomy & 3 & $1(18)$ & $1(30)$ & $1(14)$ \\
Left hepatectomy & 4 & $1(36)$ & $1(12)$ & $1(6)^{*}$ \\
& & $1(28)$ & & $1(18)^{*}$ \\
Left lobectomy & 3 & $\begin{array}{l}1(26)^{*} \\
1(12)^{*}\end{array}$ & - & $1(22)$ \\
Wedge resection & 8 & $1(38)$ & $1(25)$ & $1(30)$ \\
& & $1(36)$ & $1(36)$ & $1(18)$ \\
& & & & $1(16)$ \\
\hline
\end{tabular}

NED - No evidence of disease

Fig in parenthesis is the duration in months since liver resection

* combined with a wedge resection of the right lobe

and 25 months with a solitary metastasis [8]. Similar figures were reported by Wagner et al, who found that patients with potentially resectable metastasis had a 3 -year survival of $20 \%$ and indicated a difference in prognosis between solitary and multiple unilateral lesions, in untreated cases. However, survival rates were similar when patients with these two patterns of disease were treated by liver resection [8].

As shown by recent prospective adjuvant trials, the liver is the first and only site of failure in less than $20 \%$ of patients who experience recurrence after primary colon or rectum cancer resection [9]. Early detection of such failure can only be done by diligent screening of all patients undergoing curative surgery for colorectal cancer, since differentiation of the primary tumour does not affect, and it is controversial whether Duke's staging affects survival in patients undergoing liver resection [10]. Liver enzyme studies including estimation of serum alkaline phosphatase (SAP), gamma-glutamyl transpeptidase (GT), lactate dehydrogenase (LDH) or 5' nucleotidase are considered by some workers to be rough indicators of liver metastases [2]. We analysed SAP levels in our cases and found them elevated in only $26 \%$ of the 38 patients. CEA on the other hand is a sensitive but a nonspecific marker of tumour recurrence [11] and we found it accurate in $94 \%$ and got a false negative result in only two patients. Since a normal value of CEA does not always mean that the patient is free of disease, we follow-up all our patients with a three monthly US and a six monthly colonoscopy for the first two years and at twice the period for the next three years. Raised levels of CEA have also been shown to have prognostic importance [12].

Concomitant extrahepatic disease has an ominous prognosis and tends to be all important when liver secondaries are excised or controlled by other means. 
One third of patients who have liver-only metastasis staged by any of the presently available non-invasive imaging modalities will be found at surgery to have extrahepatic disease or metastasis to the liver too diffuse to allow surgical resection [9]. We in our experience have found such concomitant disease precluding liver resection in 20 of our $38(53 \%)$ patients. This may be because non-invasive imaging equipment is less sensitive than that available in the developed countries.

CECT is at present the most sensitive modality available to us to assess the site and size of liver metastasis. The addition of arterial portography (CTAP) to CECT is reported to increase its sensitivity from 81 to $96 \%$ and detects metastasis upto $4 \mathrm{~mm}$ in diameter [13]. Although magnetic resonance imaging (MRI) is better than CECT in evaluating local recurrence after colorectal surgery, its role in evaluating hepatic metastasis is still under evaluation [14]. CECT is an inaccurate tool for detecting extrahepatic intra-abdominal disease with a sensitivity of less than $10 \%$, and recently laparoscopic ultrasound has been added to laparoscopic screening to avoid the morbidity of an unnecessary laparotomy in such patients $[15,16]$.

The criteria and timing. for liver resection are both controversial. Opinion is divided between resecting only those patients who have less than four nodules confined to one lobe with a margin of at least $10 \mathrm{~mm}$, to resecting all metastasis provided at least one third of the liver could be preserved [10]. We have been resecting upto four nodules in the liver and performing multiple wedge resections only if such metastatic nodules are less than $2 \mathrm{~cm}$ in size. However, we found that of the patients who underwent wedge resections only $25 \%$ survived free of disease for more than 2 years. Perhaps if these patients had been observed for more time resection in them would have been precluded by the development of diffuse disease.

We have been deferring major resections for three to six months in patients with small volume resectable disease. This "trial by time" selects patients with more favourable disease and patients are offered resection if the number of secondaries remain the same even if they have increased in size. Such a policy, although advocated by most, is not concurred with universally and some workers prefer early resection in all patients since a liver metastasis may remetastasise either systemically or to lymph nodes in the hepatoduodenal ligament $[7,17]$. Propphylactic lymph node dissection of the hepato-duodenal ligament is controversial and we have not been performing it since it increases the duration of surgery without imparting a definite sur- vival benefit [18]. .

The role of surgery for bilobar metastasis. is less clear. In carefully selected patients it is not considered a contraindication of surgery. Of our 4 patients who underwent resection for bilobar disease two have survived for more than 28 months.

After resection the reported operative mortality ranges from $0-11 \%$, the 3 year survival is $44 \%$ (range $30-58$ ) [7] and the mean 5 year survival is $25 \%$ (range 16-45) [12]. The factors which effect survival both on univariate and multivariate analysis are size of metastasis (less than $7 \mathrm{~cm}$ ) and the resected margin (> $10 \mathrm{~mm}$ ). All other factors such as number of metastasis, unilateral versus bilateral disease, synchronous versus metachronous spread, tumour differentiation and Duke's stage of the primary tumour are not reported to affect survival $[10,12,19]$. We have been insisting on a resected margin of $10 \mathrm{~mm}$ and have not had any operative mortality. Although we have an overall survival of $61 \%$ and a disease free survival of $39 \%$ at a median follow-up of 28 months, our follow up is short and the total number of resections too few to reach any significant conclusion as yet.

We conclude that selected patients with hepatic secondaries from colorectal malignancy who have four or less metastasis and no demonstrable extrahepatic disease should undergo an exploratory laparotomy for a possible hepatic resection. The timing and scope of hepatic surgery must be tailored to suit the individual patient. Preoperative diagnostic laparoscopy may reduce the number of negative laparotomies by excluding patients with small sized peritoneal metastasis which cannot be picked up by US or CT. A close follow-up protocol for all patients undergoing curative surgery for colorectal cancer is essential, if such patients are to be selected early.

\section{REFERENCES}

1. Bengtsson G, Carlsson G, Hafstrom LO, Jonsson PE. Natural history of patients with untreated liver metastases from colorectal cancer. Am J Surg 1981;141:586-9.

2. Ohlsson B, Tranberg KG, Lundstedt C, Ekberg H, Hederstrom E. Detection of colorectal liver cancer: a prospective study of laboratory and imaging methods. Eur $J$ Surg 1993;159: 275-81.

3. Wood CB, Gilis CR, Blumgart LH. A retrospective study of the natural history of patients with liver metastases from colorectal cancer. Clinical Oncology 1976;2: 285-8.

4. Nordlinger B, Parc R, Delva E, Quilichini MA, Hannoun L, Huguet C. Hepatic resection for colorectal liver metastases: influence on survival of preoperative factors and surgery for recurrences in 80 patients. Ann Surg 1987;205: 256-63.

5. Scheele J, Stangl R, Altondorf-Hofmann A, Gall FP. Indicators of prognosis after hepatic resection for colorectal secon- 
daries. Surgery 1991;110:13-29.

6. Steele G Jr, Ravikumar TS. Resection of hepatic metastases from colorectal cancer: biological perspectives. Ann Surg 1989;210: 127-37.

7. Tranberg K, Bengmark S. Metastatic tumours of liver . In: LH Blumgart, editor. Surgery of the liver and biliary tract. 2nd ed. Edinburg. Churchill Livingstone. 1994; 1385-98.

8. Wagner JS, Adson MA, van Heerden JA, Adson MH, Ilstrup DM. The natural history of hepatic metastases from colorectal cancer. Ann Surg 1984;199:502-8.

9. Steele G Jr, Bleday R, Mayer RJ, Lindblad A, Petrelli N, Weaver D. A prospective evaluation of hepatic resection for colorectal carcinoma metastases to the liver: Gastrointestinal Tumour Study Group protocol 6584. J Clin Oncol 1991, 9;1105-12.

10. Rees M, Plant G, Bygrave S: Late results justify resection for multiple hepatic metastases from colorectal cancer. $\mathrm{Br}$ J Surg 1997;84:1 136-40.

11. Kemeny MM, Sugarbaker PH, Smith TJ, Edwards Bk, Shawker $T$, Vermess $M$ et al. A prospective analysis of laboratory tests and imaging studies to detect hepatic lesions. Ann Surg 1982;195:163-7.

12. Jaeck D, Bachellier P, Guigeuet $M$, Boudjema $K$, Villant JC, Balladur $\mathbf{P}$ et al. Long term survival following resection of colorectal hepatic metastases. Br J Surg 1997;84:977-80
13. Moran BJ, O'Rourke N, Plant GR, Rees M. Computed tomographic arterial portography in preoperative imaging of hepatic neoplasms. Br J Surg 1995;82:669-71.

14. Heiken JP, weyman PJ, Lee JK, Balfe DM, Picus D, Brunt $E M$ et al. Detection of focal hepatic masses: prospective evaluation with CT, delayed CT, CT during arterial portography and MR imaging. Radiology 1989;171:47-51.

15. Reed WP, Mustafa IA. Laparoscopic screening of surgical candidates with pancreatic cancer or liver tumours. Surg Endosc 1997;1:12-4.

16. Barbot DJ, Marks JH, Feld RI, Liu JR, Rosato FE. Improved staging of iver tumours using laparoscopic intraoperative ultrasound . J Surg Oncol 1997;64: 67-73.

17. August DA, Sugarbaker PH, Schneider PD. Lymphatic dissemination of hepatic metastases : implications for the follow up and treatment of patients with colorectal cancer. Cancer 1985;55: $1490-4$.

18. Beckurts KTE, Holscher AH, Thorban ST, Bollsschwiler E, Siewert JR. Significance of lymph node involvement at the hepatic hilum in the resection of colorectal liver metastases. Br J Surg 1997;84:1081-4.

19. Shirabe K, Takenaka K, Gion T, Fujiwara Y, Shiwada Y, Yanaga $\mathrm{K}$ et al. Analysis of prognostic risk factors in hepatic resection for metastatic colorectal carcinoma with special reference to the surgical margin. Br J Surg 1997;84:1077-80. 